\title{
Regional road traffic management in The Netherlands
}

\author{
J. Vrancken ${ }^{1}$, F. Ottenhof ${ }^{2} \&$ M. Soares ${ }^{1}$ \\ ${ }^{I}$ Delft University of Technology, The Netherlands \\ ${ }^{2}$ Trinité Automatisering B.V., The Netherlands
}

\begin{abstract}
This paper describes an approach to road traffic management (TM) at the network level. The paper is based on the experiences with the HARS system in and around Alkmaar, The Netherlands. This system is meant to integrate the traffic management on the urban, provincial and motorway networks near Alkmaar. The approach consists of a combination of top-down, scenario-based TM and bottom-up TM, based on communicating network elements (road segments and junctions). The paper argues that both ways have capabilities and limitations and that the best results can be achieved in combining the two and in achieving synergy between them.
\end{abstract}

Keywords: road traffic management, network management, regional traffic management, multi-agent control.

\section{Introduction}

Traffic management (TM) is applied in congested areas worldwide. Its goals are improving network performance, safety, travel time reliability and drivers' comfort, while reducing congestion and air pollution by road traffic. The oldest and best known TM-measure is the traffic signal, managing traffic at a crossing of roads. Currently, many more measures exist, but virtually all of these are local measures, with a scope in the order of a few hundred meters.

There are a number of reasons that make it desirable to take larger parts of a network into account when doing TM. First of all, it is often observed that a congestion problem dealt with using local measures without taking the network context into account, is only shifted to some other place. Secondly, a number of the criteria that express network performance, are criteria at the network level, 
such as travel times, number of car-kilometers produced by the network, or the car-hours spent in the network during a chosen period. This holds both from the administrator's and the driver's point of view (number of car-kilometers produced, travel time). It is not likely that a purely local approach to TM is the best way to meet these criteria. A third reason stems from the ambition in TM to work proactively, that is, to prevent problems rather than to solve problems after their occurrence. This makes it necessary to look into the future. It doesn't make sense to do this locally, f.i. for a single crossing. What will happen in half an hour at that crossing, depends on what happens elsewhere in the network.

This means that there is a need for network management, the management of traffic considering larger parts of a network than in traditional traffic management.

There is quite a bit of experimentation with network management $[1,10]$, for instance with phased traffic lights or belt road management, but nevertheless, this kind of TM is still in its infancy [5]. This has several causes, the most important of which is probably the chicken-and-egg dependency between the knowledge of how to do it and the required technical means: without the necessary equipment along the roads and in the traffic management centres, it is hard to experiment and to find out how network management can best be done. On the other hand, as long as there is no established and proven approach to network management, it is hard to make the high investments for the necessary equipment.

By the very complexity of network management, simulation, as an alternative to real life experiments, has only a limited value. The presence of human intelligence in the process (the drivers) makes it hard to obtain realistic simulations, especially when various basic input parameters to the simulation cannot be measured in the real world.

\section{Network management}

Network management of road traffic means that the traffic manager does not look at just one crossing or one road segment but that he takes a larger portion of the network into account, ranging from a very small network (for instance two adjacent crossings) to the complete network of a country or even a continent. Looking at a large network, it obviously doesn't make sense to take all the details into account of each crossing and each road segment, so in network management, traffic managers distinguish different levels in a network, from the very local to the very large [12]. At each level, except the highest, the network is divided into parts. Each level has its own objectives, measures and data collection. All levels, except the lowest, have as a task to coordinate the parts of the network at the adjacent lower level. This is perfectly analogous to the administration of a country: there is a national administration and the country is partitioned into a number of states or provinces, each with its own jurisdiction. Each province is partitioned into a number of municipalities or districts, of which each has also its own jurisdiction. 
At each level, there are basically two approaches to traffic management: the top-down and the bottom-up approach. Top-down means that there is one instance, usually a traffic management centre, that takes the management decisions. Key elements in this approach, as it is implemented in the Netherlands, are a reference framework and scenarios [2,3]. The reference framework consists of target figures for each network element in terms of desired maximum travel times, throughputs, intensities, speeds, etc. Scenarios are coherent series of TM-measures, to be executed in response to predefined traffic patterns (morning rush hour, weekend exodus, incidents, etc.). The main limitation of the top-down approach is that it can handle only situations with limited complexity. Only relatively few traffic patterns can be handled by an existing scenario but a sizeable network has a very large number of different situations. With increasing network size, the number of different situations increases rapidly, which makes the top-down approach badly scalable.

The limitations of the top-down approach imply that there is room for additional approaches. One such approach is bottom-up traffic management $[7,8]$. We consider this approach as an addition and not as an alternative to the top-down approach. The characteristics of the bottom-up approach are strongly different from the top-down approach, which makes it likely that both approaches will always be complementary and not alternatives that make the other approach superfluous.

The essence of the bottom-up approach is that one has a large number of items or agents [11] in the network that can take traffic management decisions, be it often on a very small scale (single crossing or even a single vehicle). The intended network effect has to emerge out of the local actions of many agents $[13,14]$. There are basically two types of candidates for the agents in road traffic management. The best known candidates are the vehicles. They are an important category and its importance is even increasing by the growing presence of computing and communicating devices inside vehicles (navigators, mobile phones with a GPS connection, PDA's etc.). Nowadays these devices are more and more provided with up-to-date traffic information. From the viewpoint of TM operators however, not so much can be done with these devices. They are under the control of individual drivers and they are being applied more or less exclusively for the user optimum instead of the system optimum. The only way to influence these devices would be by influencing the dynamic traffic information provided, but then competing service providers will appear and reduce the effectiveness of this approach.

There is a different category of agents that is much more useful for TM operators, namely the network elements. A number of elements are listed in the layered model [6] of network geometry (left most column) in table 1. The Cross Sections layer is used only in data collection (speeds and intensities are cross section properties). The lowest layer used in control is the Links and Nodes layer.

Above that layer, several kinds of composite objects can be distinguished, which in principle all may serve as agents in a bottom-up approach, but here we restrict ourselves to the Links and Nodes layer. Links are segments between two 
nodes. A node is a connection point, either a crossing or a point where a carriage way splits up (f.i. exit-ramp) or where two carriageways join (f.i. entry ramp).

Table 1: $\quad$ Road network layers.

\begin{tabular}{|l|l|l|}
\hline Road Network & Control objectives & Implementation objects \\
\hline Network & Overall network perform. & Network manager \\
\hline Origin-Destination pairs & Performance of OD-traffic & OD-manager \\
\hline Routes & Performance of a single $\mathrm{r}$. & Route-manager \\
\hline Links, Nodes & Performance of link/node & Link and node manager \\
\hline Cross Sections & & \\
\hline
\end{tabular}

In the bottom-up approach described here, the communicating agents are the second level objects: links and nodes measure their traffic state continuously and send each other messages when some of the measured parameters threatens to go out of the bounds set by the reference framework. The messages are in essence service requests, such as "reduce inflow" or "increase outflow". It is crucial here to distinguish between services and measures [2]. A service, such as "reduce inflow" may be implemented by a variety of concrete measures, depending on what the addressed link or crossing has available (shortening of green times, signaling a lower speed, closing a lane, etc.).

The agents can also be used for efficient data collection, both for basic data items that are directly measured, such as speed and intensity, and for the computation of derived data items, such as split rates at a crossing [7].

The main limitation of the bottom-up approach is the difficult relationship between the basic program running in the agents, and the intended, emergent network effect. Typical effects to be achieved by bottom-up TM are spreading of traffic over the network and the timely prevention of overloading of network elements. To obtain these effects requires quite a bit of experimentation and tuning of the basic programs in the agents. But once these effects are obtained, the approach has much better scalability than the top-down approach, both in terms of the network covered as in terms of new functions or new optimization criteria.

\section{Regional Traffic Management}

In The Netherlands, as in many other countries, there is an historical hurdle to network management. Traditional traffic management was connected to, or done by the same instance as the building and maintenance agency. Building and maintenance was and is organized per type of network: the motorway network, the urban network and the provincial or rural network. The different characteristics of the different networks from the point of view of building and maintenance make this subdivision reasonable, but from the point of view of $\mathrm{TM}$, and especially network management, it is less suitable. Obviously, within one region the different networks have strong interaction at each point where the networks meet, such as entry and exit ramps of a motorway, whilst the interaction between remote parts of for instance the motorway network, is hardly 
significant. This historical hurdle has lead to the development of Regional Traffic Management (RTM), also called Sustainable Traffic Management $[2,3]$. RTM aims at considering regions in the whole road network, and doing traffic management on all of the different types of networks in a region. It focuses especially on the interaction between the different types of networks, instead of ignoring these. RTM also aims at proactive network management and it stresses the need for cooperation between the different administrations involved in traffic management in the region. RTM does not aim at replacing all the different traffic management authorities in a region by a single authority. That would involve issues quite different from traffic management. RTM only stresses the importance of proper cooperation at the interface points of the different types of networks, and that decisions at these interface points should be taken with the complete network of the region in mind.

Currently RTM is under consideration in a number of regions in The Netherlands [1,10], but most of these projects are still in the planning phase. The Alkmaar area (fig. 1) is the first region where RTM has actually been implemented.

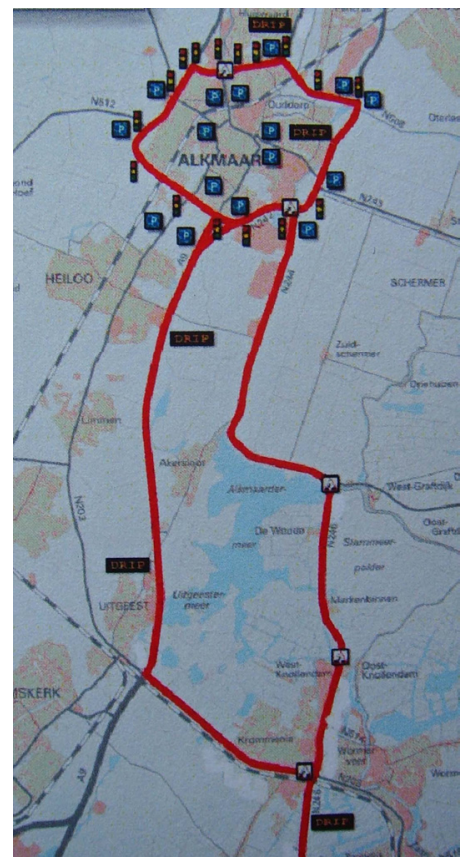

Figure 1: The network managed by HARS.

\section{The HARS system}

In fig. 1, the red roads form the network that is being managed by HARS [8] (Het Alkmaar RegelSysteem, which is Dutch for: the Alkmaar control system) in accordance with the RTM approach. 
It involves motorway segments (of the A9) as well as parts of the urban network of Alkmaar and of the provincial network around Alkmaar. The area was chosen because of frequent congestion problems. The available actuators are traffic signaling systems at crossings and DRIPs (dynamic route information panels). The system combines top-down TM from a traffic management centre, using a small number of scenario's and a traffic simulator, with bottom-up TM using nodes and links as agents. The traffic simulator can look half an hour into the future. This ensures some degree of proactive traffic management (preventing problems rather than solving them). Most links and nodes are provided with their own sensors (mostly induction loops), but those that are not have their data supplied by the simulator.

The system has been installed recently. It is currently being tuned and teething problems are being handled, so it is still too early for reliable measurements of its effects. First impressions however are promising.

\section{Conclusions}

Currently, TM in The Netherlands and in many other countries is mainly using local measures and it is done per type of network (motorway, rural, urban). Several reasons make it desirable to take larger parts of a network into account when doing TM. This is called network management. Due to the strong interaction between the different types of network at their interfacing points, network management can only be done if the whole road network of a region is taken into account. This is called Regional Traffic Management (RTM).

Due to several reasons, notwithstanding many experiments with network management, we observe that it is still in its infancy. Most of the projects in the Netherlands that experiment with RTM, are still in their planning phase. Only the HARS system at Alkmaar has (recently) been implemented and installed on the network.

The approach to network management, as applied in the HARS system, has three essential characteristics:

- Several levels are distinguished in the network, ranging from the very local (one crossing, one road segment) to the complete network of all roads in the region.

- Top-down TM is applied by means of a reference framework, scenarios and a predictive traffic simulator.

- Bottom-up TM is applied, using crossings and links between crossings as the communicating agents.

The two approaches to TM are not alternatives to each other but are considered complementary. Both have strong points and weaknesses. The combination offers the best prospects for doing network management effectively.

The interaction between the top-down and the bottom-up approach is found not only in control but also in data collection. 


\section{Future research}

Two main questions remain for future research. The first is about the relationship between emergent network effects and agent behavior in the bottom-up approach to TM. A better insight has to be obtained, on the one hand, into the emergent effects of a given agent behavior, and on the other hand, into the required agent behavior for a given desired network effect to emerge.

The second question is about the effects and the possible synergy of vehicles as agents. Vehicles are increasingly equipped with communicating and computing devices, used for trip optimisation from the driver's perspective. This will undoubtedly have an effect on network performance. It will be an important question how the TM approaches described above will cope with this crucial new development and how they can be made synergic.

\section{Acknowledgements}

This research was supported by the Next Generation Infrastructures Foundation and the Next Generation Infrastructures Research Centre, both situated in Delft, The Netherlands.

\section{References}

[1] H. Taale: Regional Traffic Management - Method and Tool, Proceedings of IFAC 2006, Delft.

[2] Rijkswaterstaat (2003): Handbook Sustainable Traffic Management, AVV Transport Research Centre.

[3] Rijkswaterstaat (2004): The Application of Sustainable Traffic Management in the Netherlands - An Overview, AVV Transport Research Centre (in Dutch).

[4] J.L.M. Vrancken: A coordination algorithm for road traffic control measures, Proceedings ICNSC2005, Tucson, March 2005.

[5] J.L.M. Vrancken, O.C. Krüse: Intelligent Control in Networks, The Case of Road Traffic Management, IEEE-ICNSC2006, Ft. Lauderdale, April 2006.

[6] J.L.M. Vrancken: Layered Models in Standardization, 2006, SMC2006, Taiwan.

[7] J.L.M. Vrancken, O.C. Krüse, Soares, M.S.: Architecture for Distributed Traffic Control, ITS World Congress 2006, London.

[8] J.L.M. Vrancken, F. Ottenhof, A Bottom-up approach to Implementing Sustainable Traffic Control, Preprints of the 11th IFAC Symposium on Control in Transportation Systems, August 2006.

[9] Lukszo, Z., L. Ferreira, J. Vrancken: Decision making in transport infrastructures, SMC2006.

[10] H.J. Stoelhorst, F. Middelham, J.L.M. Vrancken: State of the Art of Regional Traffic Management Planning in the Netherlands, Proceedings 
282 Urban Transport XIII: Urban Transport and the Environment in the 21st Century

of the 11th IFAC Symposium on Control in Transportation Systems, August 2006.

[11] M.S. Soares, J.L.M. Vrancken, A Multi-agent Distributed Architecture for Road Traffic Control, ITS Europe Congress, 2007, Aalborg, Denmark, accepted.

[12] J.L.M. Vrancken, M. Soares: Multi-level Control of Networks, the Case of Road Traffic Control, IEEE-SMC, Montreal, 2007, submitted.

[13] S. Johnson: Emergence, the Connected Lives of Ants, Brains, Cities, and Software, 2002.

[14] J.H. Holland: Emergence: From Chaos to Order, 2000. 